\section{ENSINO DE HÁBITOS POSTURAIS EM CRIANÇAS: HISTÓRIA EM QUADRINHOS VERSUS TEATRO DE FANTOCHES}

\author{
Postural education in children: comics versus puppet theatre \\ Enseñanza de los hábitos posturales en niños: historietas \\ versus teatro de marionetas
}

\section{RESUMO}

Objetivo: Comparar a efetividade das histórias em quadrinhos e do teatro de fantoches na aprendizagem de hábitos posturais em crianças na idade escolar. Métodos: Estudo de campo, de caráter longitudinal e quantitativo, realizado em uma escola privada localizada no município de Caucaia-CE-Brasil, durante o período de fevereiro a novembro de 2012. Os alunos foram divididos igualmente, de forma aleatória, em dois grupos: Grupo A, no qual as crianças receberam informações através da utilização da história em quadrinhos; e Grupo B, no qual as crianças receberam informações através da apresentação de um teatro de fantoches. As avaliações ocorreram em dois momentos: antes e 2 meses após as intervenções educativas. As variáveis estudadas foram a avaliação da postura corporal e o conhecimento dos hábitos posturais corretos. Resultados: Participaram do estudo 52 crianças de ambos os gêneros. Observamos que tanto as crianças do Grupo A como as do Grupo B aprenderam sobre os hábitos posturais corretos. No Grupo A, apenas $23 \%(n=6)$ tiveram alguma dificuldade no entendimento das imagens e $58 \%(\mathrm{n}=15)$ afirmaram que melhoraram a forma de andar e sentar. No Grupo B, 12\% $(n=13)$ relataram que tiveram alguma dificuldade no entendimento da linguagem e $50 \%(n=13)$ que melhoraram a forma de andar e sentar. Conclusão: Os achados deste estudo revelaram que as duas formas de estratégia mostraram-se efetivas para ensinar e fixar conceitos sobre os hábitos posturais corretos.

Descritores: Educação em Saúde; Postura; Prevenção Primária.

\section{ABSTRACT}

Objective: To compare the effectiveness of comics and puppet theatre in postural education in school-age children. Methods: Field study of longitudinal and quantitative approach, carried out in a private school located in the city of Caucaia, Ceará, Brazil, in the period from February to November 2012. The students were equally divided, at random, into two groups: Group A, in which the children received information with use of comics; and Group $B$, in which the children received information through a puppet theatre presentation. The evaluation occurred at two moments: prior to and two months after the educational interventions. The studied variables were the body posture assessment and the knowledge of correct postural habits. Results: The study comprised 52 children of both genders. It was observed that children of both group A and group B learned about the correct postural habits. In Group A, only 23\% $(n=6)$ had some difficulty in understanding the images and 58\% $(n=15)$ said they improved their way of walking and sitting. In group $B, 12 \%(n=3)$ reported some difficulty in understanding the language and $50 \%(n=13)$ stated they improved their way of walking and sitting. Conclusion: The findings of this study revealed that the two forms of strategies were effective for teaching and retaining concepts on correct postural habits.

Descriptors: Health Education; Posture; Primary Prevention.
Artigo Original
1) Centro Universitário Estácio do Ceará Fortaleza (CE) - Brasil

2) Universidade Federal do Ceará - UFC Fortaleza (CE) - Brasil

Recebido em: 17/07/2013

Revisado em: 26/11/2013 Aceito em: 12/6/2014 


\section{RESUMEN}

Objetivo: Comparar la eficacia de las estrategias de las historietas versus teatro de marionetas en el aprendizaje de hábitos posturales en los niños en edad escolar. Métodos: Estudio de campo de carácter intervencionista y longitudinal con el análisis cuantitativo de los resultados realizados en una escuela privada situada en el municipio de Caucaia-CE-Brasil durante el periodo de febrero a noviembre de 2012. Los estudiantes se dividieron por igual en dos grupos: grupo A en el que los niños recibieron información a través de la utilización de historietas y los niños del grupo $B$ que recibieron información a través de la presentación de un teatro de marionetas. Las evaluaciones se dieron en dos momentos: antes y dos meses después de las intervenciones educativas. Las variables estudiadas fueron la evaluación de la postura corporal y el conocimiento de los hábitos posturales correctos. Resultados: El estudio incluyó a 52 niños de ambos sexos, se observó que los niños de los grupos $A$ y $B$ aprendieron acerca de los hábitos posturales correctos. En el grupo A el 23\% $(n=6)$ tuvieron dificultad en la comprensión de las imágenes y el $58 \%(n=15)$ indicaron que mejoró la forma de caminar y sentarse correctamente. En el grupo B, el 12\% $(n=3)$ dijeron que tenían cierta dificultad en la comprensión del lenguaje y el 50\% $(n=13)$ relató mejora de la forma de caminar y sentarse correctamente. Conclusión: Los hallazgos de este estudio mostraron que los dos tipos de estrategias son eficaces en el sentido de los conceptos de enseñanza y establecimiento de hábitos posturales correctos.

Descriptores: Educación en Salud; Postura; Prevención Primaria.

\section{INTRODUÇÃO}

A postura corporal da população remete-nos a uma preocupação crescente, principalmente quando se leva em consideração o estilo de vida a que nos submetemos hoje ${ }^{(1)}$. Essa preocupação tem início desde o período da infância - a prevalência global de escoliose está em torno de $1 \%$ a $2 \%$, sendo a escoliose idiopática em adolescentes a mais comum $^{(2)}$.

Hábitos posturais inadequados adotados por crianças, em casa e/ou na escola, podem originar um desequilíbrio na musculatura do corpo, produzindo alterações posturais. Os problemas físicos que têm início na fase de crescimento constituem fator de risco para disfunções da coluna vertebral irreversíveis na fase adulta. Por isso, é importante a detecção precoce e a prevenção desses problemas, associadas a ações educativas quanto aos hábitos posturais corretos, além de intervenções fisioterapêuticas, com o objetivo de prevenir as dores na coluna vertebral dos adolescentes ${ }^{(1,3)}$.

A educação em saúde é um processo de ensinoaprendizagem que visa a prevenção doenças e a promoção de saúde para a construção de condições de vida saudável ${ }^{(4)}$.
A estratégia de promoção da saúde é ampla e, na escola, envolve vários atores sociais, como alunos, professores, coordenadores, donos de cantinas e pais ou responsáveis, permitindo uma abordagem dialógica da produção do conhecimento no ambiente escolar ${ }^{(5)}$. Programas de intervenção comunitários que versam sobre promoção de saúde e prevenção de doenças e agravos têm sido utilizados por diversos países no mundo desde a década de 1970, tendo como alicerces a identificação e o enfrentamento dos determinantes do processo saúde-doença, na busca constante de reduzir os fatores de risco ${ }^{(6)}$.

Visando otimizar o processo de ensino e motivação para o aprendizado de conceitos relacionados a hábitos posturais, várias técnicas de aprendizado das posturas corretas e incorretas são utilizadas, como videoteipes, circuitos práticos, dramatização, história em quadrinhos, cartazes, cartilhas, demonstração com bonecos, dentre outras. A facilidade com que esses instrumentos comunicam conhecimentos científicos está relacionada ao fato de que eles transmitem informações de forma atrativa e divertida, facilitando a memorização de conceitos e traduzindo com clareza a mensagem que se deseja $\operatorname{comunicar}^{(7)}$.

O lúdico pode ser usado como um recurso de comunicação na vinculação de informações de saúde, pois ele desperta a criatividade e mantém a atenção dos participantes, estimulando com maior facilidade a participação ativa $^{(7,8)}$. É um elemento essencial no trabalho com a criança, afinal, a infância é a etapa do desenvolvimento humano em que o indivíduo passa a se conhecer como um ser social e a formar uma concepção de mundo baseada na realidade em que vive.

Dessa forma, este estudo surgiu da necessidade de identificar os possíveis hábitos posturais adotados pelos estudantes em idade escolar e utilizar meios de educação em saúde interessantes e didáticos, com o intuito de se tentar minimizar a alta incidência de afecções posturais no adulto. Para isso, faz-se necessário um trabalho de base abrangente, atuando principalmente no plano preventivo e educacional, possibilitando a mudança de hábitos inadequados na fase de crescimento.

Sendo assim, este estudo teve como objetivo comparar a efetividade das histórias em quadrinhos e do teatro de fantoches na aprendizagem de hábitos posturais em crianças na idade escolar.

\section{MÉTODOS}

Estudo de campo de caráter interventivo e longitudinal, com estratégia de análise quantitativa dos resultados, realizado em uma escola privada localizada no município de Caucaia-CE-Brasil, durante o período de fevereiro a novembro de 2012 . 
Foram considerados sujeitos do estudo as crianças de ambos os gêneros com idade entre 5 e 10 anos, visto que há predominância de crianças na escola dentro dessa faixa etária, totalizando $80 \%$ de crianças por cada faixa etária (aproximadamente de 8 a 10 crianças por cada faixa etária). Foram excluídas as crianças que apresentaram problemas neurológicos, deformidades ortopédicas e amputações, por serem disfunções que dificultavam a compreensão das perguntas ou influenciavam a postura.

O estudo foi iniciado após a assinatura do termo de consentimento livre e esclarecido pelos pais ou responsáveis. Em seguida, os alunos foram divididos igualmente, na totalidade e por faixa etária, em dois grupos:

Grupo A: Crianças que receberam informações teóricas sobre postura correta e relaxamento, acrescentando a utilização da história em quadrinhos (Figura 1).

Grupo B: Crianças que receberam informações teóricas sobre postura correta e relaxamento, acrescentando a apresentação de teatro de fantoches com bonecos (Dra. Ana, Tiago e Julinha), confeccionados de acordo com o tema relacionado aos hábitos posturais (Figura 1).

A

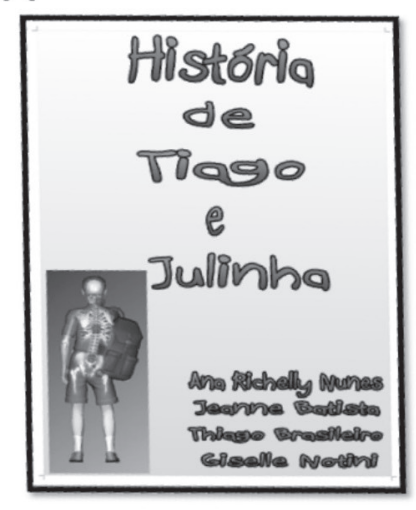

$\mathrm{B}$

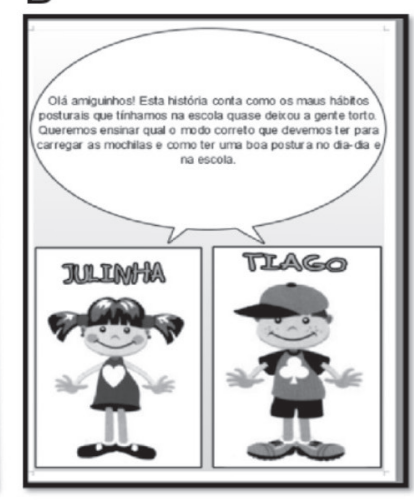

C

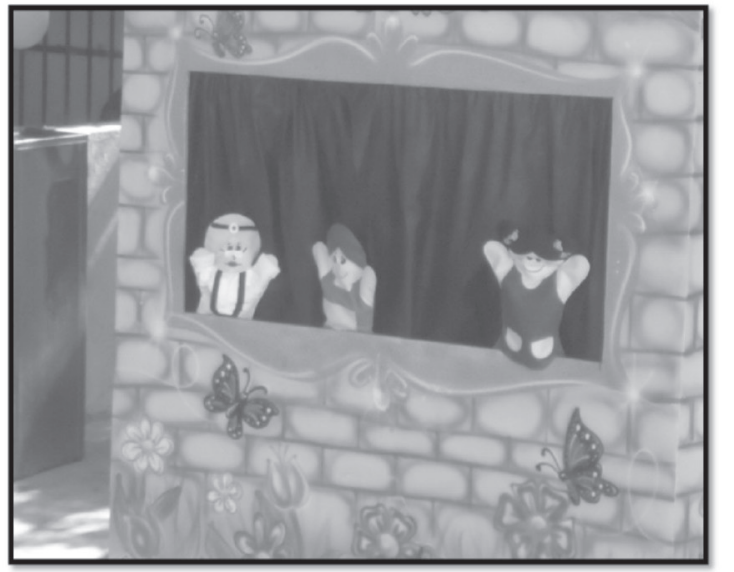

Figura 1 - Estratégia de ensino em saúde sobre os hábitos posturais corretos. A e B: História em quadrinhos; C: Teatro de fantoches.
A coleta de dados foi realizada por meio de uma ficha de avaliação postural na vista anterior, lateral e posterior ${ }^{(9,10)}$. Para tanto, as crianças usaram roupas de ginástica ou roupas leves (shorts, camisetas, tops). Logo após, responderam a um questionário ilustrativo, elaborado pelos autores, o qual objetivou o conhecimento dos hábitos posturais adotados pelas crianças em diferentes posições, sendo composto por 14 perguntas objetivas e dividido em 6 categorias que englobam as seguintes situações: postura da coluna, quadris e pés durante a posição sentada; postura da cabeça, coluna e pés na posição em pé; maneira de carregar mochilas; maneira de abaixar; maneira de mudar objetos de lugar; $\mathrm{e}$ o jeito de dormir.

Após dois meses da apresentação das estratégias educativas, foi realizada na escola uma nova avaliação individual com as crianças pesquisadas inicialmente, a fim de observar comparativamente o conhecimento dos hábitos posturais corretos entre os dois grupos em estudo e a opinião delas sobre as estratégias utilizadas.

Os dados foram analisados através do programa SPSS, versão 20 , adotando nível de significância de $5 \%(\mathrm{p}<0,05)$. Utilizou-se análise de variância (ANOVA), teste $t$ de Student e Qui-quadrado, de acordo com o teste de normalidade Kolmogorov-Smirnov (KS).

O estudo foi aprovado pelo Comitê de Ética em Pesquisa do Centro Universitário Estácio do Ceará (Parecer $n^{\circ}$ 02/12) em obediência a Resolução 466/12 do Conselho Nacional de Saúde.

\section{RESULTADOS}

Participaram do estudo 52 crianças $(\mathrm{n}=10$ na idade de 5,7 e 9 anos; $n=8$ na idade de 6 e 8 anos e $n=6$ na idade de 10 anos) de ambos os gêneros, sendo $46 \%(n=12)$ do sexo masculino e $54 \%(n=14)$ do sexo feminino no Grupo A, e $57 \%(n=15)$ do sexo masculino e $42 \%(n=11)$ do sexo feminino no Grupo B, com médias de idade de 7,15 $( \pm 1,64)$ anos e 7,26 $( \pm 1,82)$ anos, respectivamente, não apresentando diferenças estatísticas quando comparadas as médias das idades ( $\mathrm{p}>0,05)$.

Com relação ao Grupo A, foi constatado que $73 \%$ $(n=19)$ das crianças não relataram sentir dores na região das costas e $11 \%(n=3)$ das que sentiam dores nas costas relataram que costumavam senti-las às vezes, mesmo quando deitadas. Nas crianças do Grupo B, 42\% $(n=11)$ não sentiam dores nas costas, porém, $27 \%(\mathrm{n}=7)$ relataram senti-las às vezes, quando utilizavam o computador.

A Tabela I corresponde à avaliação da postura nas posições anterior, lateral e posterior dos grupos A e B. Observa-se que as crianças apresentaram uma maior diferença percentual entre os grupos apenas na simetria na 
Tabela I - Distribuição das crianças participantes a avaliação postural na vista anterior, lateral e posterior. Fortaleza-CE, 2012.

\begin{tabular}{|c|c|c|c|c|c|}
\hline \multirow[t]{2}{*}{ Avaliação Postural } & \multicolumn{2}{|c|}{$\begin{array}{c}\text { Grupo A } \\
\text { história em quadrinhos }\end{array}$} & \multicolumn{2}{|c|}{$\begin{array}{c}\text { Grupo B } \\
\text { teatro de fantoches }\end{array}$} & \multirow[t]{2}{*}{$p$} \\
\hline & n & $\%$ & $\mathrm{n}$ & $\%$ & \\
\hline \multicolumn{6}{|l|}{ Anterior } \\
\hline Posição da cabeça & & & & & \multirow{4}{*}{0,27} \\
\hline Centralizada & 22 & 84 & 22 & 84 & \\
\hline Lateralizada à D & 2 & 8 & 2 & 8 & \\
\hline Lateralizada à E & 2 & 8 & 2 & 8 & \\
\hline \multicolumn{6}{|l|}{ Ombros } \\
\hline Simétricos & 18 & 69 & 23 & 88 & \multirow{3}{*}{0,35} \\
\hline $\mathrm{D}$ mais alto & 1 & 4 & 0 & 0 & \\
\hline E mais alto & 7 & 27 & 3 & 12 & \\
\hline \multicolumn{6}{|l|}{ Crista Ilíaca } \\
\hline Simétria & 23 & 88 & 25 & 96 & \multirow{3}{*}{0,92} \\
\hline D mais alta & 2 & 8 & 0 & 0 & \\
\hline E mais alta & 1 & 4 & 1 & 4 & \\
\hline $\begin{array}{l}\text { Joelho } \\
\text { Normal } \\
\text { Genuvaloo }\end{array}$ & 25 & $\begin{array}{c}96 \\
4\end{array}$ & $\begin{array}{c}22 \\
2\end{array}$ & $\begin{array}{c}84 \\
8\end{array}$ & \multirow[b]{2}{*}{0,91} \\
\hline $\begin{array}{l}\text { Genuvalgo } \\
\text { Genuvaro }\end{array}$ & $\begin{array}{l}1 \\
0\end{array}$ & 0 & $\begin{array}{l}2 \\
2\end{array}$ & $\begin{array}{l}8 \\
8\end{array}$ & \\
\hline \multicolumn{6}{|l|}{$\underline{\text { Lateral }}$} \\
\hline \multicolumn{6}{|l|}{ Coluna Cervical } \\
\hline Normal & 25 & 96 & 26 & 100 & \multirow{4}{*}{0,79} \\
\hline Hiperlordose & 0 & 0 & 0 & 0 & \\
\hline Retificada & 1 & 4 & 0 & 0 & \\
\hline Hipercifose & 0 & 0 & 0 & 0 & \\
\hline \multicolumn{6}{|l|}{ Coluna Dorsal } \\
\hline Normal & 26 & 100 & 26 & 100 & \multirow{3}{*}{0,74} \\
\hline Hipercifose & 0 & 0 & 0 & 0 & \\
\hline Retificada & 0 & 0 & 0 & 0 & \\
\hline \multicolumn{6}{|l|}{ Flexão Anterior } \\
\hline Sem Gibosidade & 26 & 100 & 24 & 92 & \multirow{3}{*}{1} \\
\hline Gibosidade à D & 0 & 0 & 1 & 4 & \\
\hline Gibosidade à E & 0 & 0 & 1 & 4 & \\
\hline \multicolumn{6}{|l|}{ Posterior } \\
\hline \multicolumn{6}{|c|}{ Proeminência Escapular } \\
\hline Simétrica & 24 & 92 & 25 & 96 & \multirow{3}{*}{0,92} \\
\hline Lado D & 0 & 0 & 0 & 0 & \\
\hline Lado E & 2 & 8 & 1 & 4 & \\
\hline \multicolumn{6}{|l|}{ Triângulo de Thales } \\
\hline Simétrico & 23 & 88 & 23 & 88 & \multirow{3}{*}{0,28} \\
\hline Maior à D & 2 & 8 & 1 & 4 & \\
\hline Maior à E & 1 & 4 & 2 & 8 & \\
\hline
\end{tabular}

$\mathrm{D}=$ direita; $\mathrm{E}=$ esquerda. 
Tabela II - Distribuição das crianças participantes quanto aos hábitos posturais corretos. Fortaleza-CE, 2012.

\begin{tabular}{llccc}
\hline $\begin{array}{l}\text { Hábitos } \\
\text { Posturais }\end{array}$ & Posição Correta & $\begin{array}{c}\text { Grupo A } \\
\text { história em quadrinhos } \\
\text { (\%) }\end{array}$ & $\begin{array}{c}\text { Grupo B } \\
\text { teatro de fantoches } \\
\text { (\%) }\end{array}$ & $\boldsymbol{p}$ \\
\hline Sentada & Costas & 54 & 42 & 0,33 \\
& Bumbum & 84 & 58 & 0,78 \\
& Pés & 61 & 35 & 0,71 \\
Em pé & Costas & & & 0,28 \\
& Pés & 64 & 65 & 0,49 \\
& & 65 & 61 & 0,84 \\
& Sentado no computador & 58 & 50 & 0,64 \\
& Transporte de mochila & 58 & 54 & 0,24 \\
& Pegar objetos no chão & 61 & 65 & $0,03 *$ \\
\hline
\end{tabular}

*p $<0,05$.

Tabela III - Opinião e conhecimento das crianças participantes após a utilização das duas formas de estratégia de educação em saúde. Fortaleza-CE, 2012.

\begin{tabular}{|c|c|c|c|}
\hline Pergunta & $\begin{array}{c}\text { Grupo A } \\
\text { história em quadrinhos }\end{array}$ & $\begin{array}{l}\text { Grupo B } \\
\text { teatro de fantoches }\end{array}$ & $p$ \\
\hline \multicolumn{4}{|l|}{ Gostou da iniciativa? } \\
\hline $\operatorname{Sim}$ & $100 \%(n=26)$ & $100 \%(n=26)$ & - \\
\hline Não & 0 & 0 & \\
\hline \multicolumn{4}{|l|}{ Entendeu as imagens? } \\
\hline Sim & $77 \%(n=20)$ & $88 \%(n=23)$ & \multirow{3}{*}{0,20} \\
\hline Não & $4 \%(n=1)$ & 0 & \\
\hline Algumas & $19 \%(n=5)$ & $12 \%(n=3)$ & \\
\hline \multicolumn{4}{|l|}{ O que não entendeu? } \\
\hline Como sentar & 0 & $4 \%(n=1)$ & \multirow{3}{*}{$0,04^{*}$} \\
\hline Como andar corretamente & $12 \%(n=3)$ & 0 & \\
\hline Como carregar a mochila & $4 \%(n=1)$ & 0 & \\
\hline \multicolumn{4}{|l|}{ Entendeu a linguagem? } \\
\hline $\operatorname{Sim}$ & $77 \%(\mathrm{n}=20)$ & $88 \%(n=23)$ & \multirow{3}{*}{0,66} \\
\hline Não & $4 \%(n=1)$ & $4 \%(n=1)$ & \\
\hline Um pouco & $19 \%(n=5)$ & $8 \%(n=2)$ & \\
\hline \multicolumn{4}{|l|}{ O que mudou no seu comportamento? } \\
\hline Aprendeu a sentar e andar corretamente & $58 \%(\mathrm{n}=15)$ & $50 \%(n=13)$ & \multirow{4}{*}{0,54} \\
\hline Diminuiu o peso da mochila & $12 \%(n=3)$ & $8 \%(n=2)$ & \\
\hline Começou a dobrar os joelhos para pegar objetos & $12 \%(n=3)$ & $12 \%(n=3)$ & \\
\hline Nada mudou & $18 \%(n=5)$ & $30 \%(n=8)$ & \\
\hline
\end{tabular}

$$
{ }^{*} \mathrm{p}<0,05
$$


altura dos ombros (19\%), ombro esquerdo mais alto (15\%) e joelhos normais (12\%) (Tabela I), não apresentando diferenças estatísticas quando comparado os dois grupos $(\mathrm{p}>0,05)$.

Com relação aos hábitos posturais, a Tabela II mostra o percentual das respostas corretas ao questionário sobre as 6 categorias de posturas pesquisadas. As maiores diferenças percentuais foram apresentadas no Grupo B, quanto às posturas corretas de sentar encostando o "bumbum" na cadeira (26\%) e os pés no chão $(26 \%)$, e de ficar em pé com as costas retas $(19 \%)$.

Após 2 meses, as crianças foram reavaliadas sobre a eficácia dos meios de aprendizagem. Notou-se uma semelhança entre os grupos quando todas (100\%) as crianças responderam que gostaram da iniciativa. No Grupo A, apenas $23 \%(n=6)$ tiveram alguma dificuldade no entendimento das imagens e $58 \%(\mathrm{n}=15)$ melhoraram a forma de andar e sentar. No Grupo B, $12 \%(n=3)$ tiveram alguma dificuldade no entendimento da linguagem e $50 \%$ $(n=13)$ melhoraram a forma de andar e sentar (Tabela III). As maiores diferenças percentuais entre os grupos foram no entendimento da estratégia de ensino (11\%), sendo relatada maior facilidade de entender pelas crianças do Grupo B, porém, $30 \%$ afirmaram não ter mudado o seu comportamento após as informações recebidas, não apresentando diferenças estatísticas quando comparado os dois grupos $(p>0,05)$.

\section{DISCUSSÃO}

As duas estratégias de ensino utilizadas no presente estudo mostraram-se efetivas para ensinar e fixar conceitos sobre os hábitos posturais corretos.

Esses dados vão ao encontro de outros estudos, que destacam a importância da postura adequada na infância ou a correção precoce de desvios posturais nessa fase, com o intuito de possibilitar padrões posturais corretos na vida adulta, já que esse período é o de maior importância para o desenvolvimento musculoesquelético do indivíduo, com maior probabilidade de prevenção e tratamento das alterações posturais ${ }^{(3,11,12)}$.

Portanto, se todos os padrões de postura assumidos na infância formam um padrão e se tornam inconscientes, é na infância que se deve promover a correção desses possíveis vícios posturais e a educação dos hábitos de postura nas atividades da vida diária, para que no futuro os indivíduos adultos não sofram ou pelo menos diminuam os problemas na coluna vertebral. As crianças pesquisadas apresentaram uma média de idade em torno dos 7 anos e não apresentaram desvios posturais importantes, no entanto, outros estudos com adolescentes (14 a 17 anos) já mencionam um aumento dos agravos posturais, sendo a idade, portanto, um fator diretamente relacionado ao início das alterações ${ }^{(12,13)}$. É importante também observar a associação entre postura, oclusão e ortóptica, pois muitas vezes essa conexão causal é despercebida, daí a importância de uma abordagem multidisciplinar $^{(11)}$.

A avaliação postural de crianças pode contribuir muito mais para a sociedade em que vivemos, evitando alterações na coluna vertebral, melhorando o rendimento do aluno e promovendo o seu bem-estar físico e emocional ${ }^{(14)}$.

Um estudo verificou a incidência de desvios posturais em 154 escolares da cidade de Novo Hamburgo-RS, de 6 a 17 anos. Os resultados demonstraram que, em relação à coluna vertebral, apenas $29,22 \%$ das meninas avaliadas apresentaram a coluna vertebral sem nenhum desvio, enquanto $70,78 \%$ apresentaram alguma alteração postural. Os desvios mais observados foram: anteriorização da coluna cervical e protusão de ombros ${ }^{(15)}$.

Outro estudo ${ }^{(12)}$ avaliou 1.340 escolares com média de idade de 12,7 anos e observou que a prevalência de escoliose foi de $1,4 \%$; assimetria de ombros e escápula, $6,6 \%$; assimetria antebraço e tronco, $4,0 \%$; desalinhamento da coluna vertebral, $1,9 \%$; e assimetria do triângulo de Tales, 6,4\%. Portanto, há uma divergência quanto ao percentual de alterações posturais. No presente estudo, as crianças apresentaram uma maior diferença percentual entre os grupos apenas na simetria na altura dos ombros $(19 \%)$, ombro esquerdo mais elevado (15\%) e normalidade dos joelhos (12\%), o que demonstra, ao menos em parte, homogeneidade na avaliação postural entre os 2 grupos.

Um alinhamento postural adequado depende de vários fatores. Com isso, o trabalho na área de reeducação postural necessita de uma atuação multiprofissional e multidisciplinar para a obtenção do sucesso almejado. Os pais, professores de sala de aula, diretores das escolas, educadores físicos, médicos, fisioterapeutas, psicólogos, dentre outros, todos têm a função de observar e orientar adequadamente crianças e adolescentes em relação às atitudes posturais cotidianas, com o intuito de tornar o desenvolvimento mais harmônico e promover mudanças no ambiente escolar ${ }^{(4,7,11)}$.

Após a aplicação das estratégias de ensino em saúde, foi observado que tanto as crianças do Grupo A como as do Grupo B aprenderam sobre hábitos posturais corretos com as metodologias propostas, que preconizaram a utilização da criatividade e do lúdico em suas abordagens, sendo, portanto, possível a disseminação do conhecimento através de novas metodologias de ensino-aprendizagem ${ }^{(7,13,16-18)}$. Apesar de poucas crianças não terem entendido sobre os hábitos posturais corretos, vale ressaltar que as crianças do Grupo B foram as que obtiveram maior facilidade de aprendizado. Isso pode ser explicado pelo fato de o teatro despertar maior atenção do público. 
O teatro pode ser uma importante ferramenta para a educação dos estudantes, uma vez que, devidamente utilizado, auxilia no desenvolvimento da criança e do adolescente como um todo, promove a socialização e a melhora da aprendizagem de conteúdos escolares. Ressaltase, ainda, que o teatro também incomoda no sentido filosófico, porque estimula o pensamento e a modificação da realidade instaurada ${ }^{(19)}$.

$\mathrm{O}$ aprendizado pode ter sido beneficiado pelo fato de que as crianças estão em idade escolar, entre 5 e 10 anos, correspondendo ao período operacional concreto, ou "idade da razão". É nessa fase que elas manifestam a capacidade de passar das premissas às conclusões. Como consequência das operações concretas, gradualmente, as crianças começam a entender, entre outras coisas, o relógio, o calendário, o tempo histórico e a distinção entre causalidade física e psicológica $^{(17)}$.

No entanto, ainda não é dada a devida importância para essa fase de crescimento e suas repercussões em longo prazo. Um estudo interessante ${ }^{(18)}$, realizado com professores de uma escola, abordou o seguinte questionamento: "Você acha que os professores da sua escola estão aptos a fazer parte desse acompanhamento programado de prevenção, diagnóstico precoce e recuperação dos agravos à saúde?". Como resposta, $63,3 \%$ informaram que não, portanto, os autores sugerem maior capacitação dos educadores, com o intuito de atender às diretrizes do Programa Saúde na Escola (PSE).

Entende-se como sendo fundamental que se inicie logo na infância uma orientação postural adequada, alertando sobre o perigo das más atitudes corporais durante a execução das atividades da vida diária e demonstrando as posturas corretas a serem utilizadas no dia a dia ${ }^{(20)}$.

Foi evidente o grau de aceitação, por parte das crianças, das duas estratégias utilizadas neste estudo, pois informaram que gostaram da iniciativa e destacaram que mudaram seus hábitos posturais, principalmente no tocante à forma de andar e se sentar. Corroborando com este achado, outro estudo realizado com escolares na cidade de GaribaldiRS obteve boa aceitação por parte das crianças e também constatou que o ato de pegar um objeto do chão de maneira correta foi bem fixado pelos alunos, assim como a postura de dormir de lado, acreditando que isso foi possível devido ao fato de terem sido realizadas demonstrações práticas dessas posturas ${ }^{(21)}$.

A fisioterapia ainda é pouco inserida no ambiente escolar, o que ocasiona maior atenção, especialmente em relação aos aspectos preventivos e de promoção da saúde $^{(3,11,21,22)}$.

O estudo teve como limitações a avaliação de uma única escola e o pequeno tamanho amostral.
Mesmo assim, o objetivo principal foi atingido, pois as crianças demonstraram boa aceitação e assimilação dos conhecimentos. Estudos posteriores mais aprofundados, com um acompanhamento até a fase adulta, são necessários para comprovar a eficácia das estratégias de ensino como formas de prevenção dos desvios posturais.

\section{CONCLUSÃO}

Os achados deste estudo revelaram que as duas formas de educação em saúde - história em quadrinhos e teatro de fantoches - mostraram-se efetivas para ensinar e fixar conceitos sobre os hábitos posturais corretos, não sendo detectadas diferenças elevadas no aprendizado e na memorização dos hábitos posturais quando comparados os dois grupos.

\section{REFERÊNCIAS}

1. Contri DE, Petrucell A, Perea DCBNM. Incidência de desvios posturais em escolares do $2^{\circ}$ ao $5^{\circ}$ ano do Ensino Fundamental. Conscientiae Saúde. 2009;8(2):219-24

2. Cabral LTB, Filho ESV, Ueno FH, Yonezaki AM, Rodrigues LMR. Avaliação da qualidade de vida em pacientes com escoliose idiopática do adolescente após tratamento cirúrgico pelo questionário SF-36. Coluna/ Columna. 2009;8(3):315-22.

3. Souza Junior JV, Sampaio RMM, Aguiar JB, Pinto FJM. Perfil dos desvios posturais da coluna vertebral em adolescentes de escolas públicas do município de Juazeiro do Norte - CE. Fisioter Pesqui. 2011;18(4): 311-6.

4. Heisler EV, Vargas MD, Ghedini QP, Nogueira QD, Rodrigues MS, Cosentino SF. Teatro de fantoches: uma estratégia para educação em saúde de escolares [acesso em 2012 Abr 1]. Disponível em: http:// ebookbrowse.com/teatro-de-fantoches-uma-estrategiapara-educacaoem- saude-de-escolares-pdf-d25691465.

5. Bizzo MLG, Leder L. Educação Nutricional nos parâmetros curriculares nacionais para o ensino fundamental. Rev Nutr. 2005;18(5):661-7.

6. Pan American Health Organization. Carmen: an initiative for integrated prevention of noncommunicable diseases in the Americas [Internet]. Washington: PAHO; 2003 [acesso em 2014 Mar 21]. Disponível em: http://www1.paho.org/English/AD/DPC/NC/ CARMEN-doc2.pdf.

7. Rampaso DAL, Doria MAG, Oliveira MCM, Silva GTR. Puppet theatre as teaching strategy: a report of the experience. Rev Bras Enferm. 2011;64(4):783-85. 
8. Luchetti AJ, Moreale VC, Parro MC. Educação em saúde: uma experiência com teatro de fantoches no ensino nutricional de escolares. CuidArte Enferm. 2011;5(2):97-103.

9. Menezes TV. Estudo comparativo do comportamento postural de bailarinas da dança clássica. [monografia de graduação]. Fortaleza: Faculdade Integrada do Ceará; 2008.

10. Meliscki GA, Monteiro LZ, Giglio CA. Avaliação postural de nadadores e sua relação com o tipo de respiração. Fisioter Mov. 2011;24(4):721-8.

11. Silvestrini-Biavati A, Migliorati $M$, Demarziani E, Tecco S, Silvestrini-Biavati P, Polimeni A, et al.Clinical association between teeth malocclusions, wrong posture and ocular convergence disorders: an epidemiological investigation on primary school children. BMC Pediatrics. 2013;13:12.

12. Nery LS, Halpern R, Nery PC, Nehme KP, Tetelbom SA. Prevalence of scoliosis among school students in a town in southern Brazil. São Paulo Med J. 2010;128(2):69-73.

13. Rebolho MCT, Casarotto RA, João SMA. Estratégias para ensino de hábitos posturais em crianças: história em quadrinhos versus experiência prática. Fisioter Pesqui. 2009;16(1):46-51.

14. Back CMZ, Lima IAX. Fisioterapia na escola: avaliação postural. Fisioter Bras. 2009;10(2):72-7.

15. Detsch C, Candotti CT. A incidência de desvios posturais em meninas de 6 a 17 anos da cidade de Novo Hamburgo. Rev Movimento. 2001;7(15):43-56.

16. Nazima TJ, Codo CRB, Paes IADC, Bassinello GAH. Orientação em saúde por meio do teatro: relato de experiência. Rev Gaúch Enferm. 2008;29(1):147-51.

17. Silva JR. A Eficácia do lúdico como método de ensino na educação infantil? [acesso em 2014 Mar 22]. Disponível em: http://www.portaleducacao.com.br/educacao/ artigos/21308/a-eficacia-do-ludico-como-metodo-deensino-na-educacao-infantil\#ixzz3E0rsYIny.
18. Braga MS, Rodrigues DCGA. A avaliação da acuidade visual do escolar no programa saúde na escola: proposta de vídeo para orientação docente. Rev Ensino Saúde Ambiente. 2014;7 (1):1-11.

19. Miranda JL, Elias RC, Faria RM, Silva VL, Felício WAS. Teatro e a escola: funções, importâncias e práticas. Rev CEPPG CESUC. 2009;11(20):172-81.

20. Carneiro FR, Vasconcelos TB, Silva JHF, Josino JB, Arcanjo GN. Análise dos hábitos posturais de crianças após a conscientização educativa. In: Anais do V Congresso Internacional de Terapia Manual. Rev Inspirar. 2012;(2 Sup 1):48.

21. Benini J, Karolczak APB. Benefícios de um programa de educação postural para alunos de uma escola municipal de Garibaldi, RS. Fisioter Pesqui. 2010;17(4):346-51.

22. Caminha BJL, Vasconcelos TB, Carneiro FR, Siqueira RJB, Cardoso KVV, Nogueira MM, et al. Análise postural de alunos da $5^{\mathrm{a}}$ série do ensino fundamental em uma escola pública de Fortaleza/CE. Rev Saúde Diálogo. 2013;4(1):6-19.

Endereço para correspondência:

Ana Richelly Nunes Rocha Cardoso

Centro Universitário Estácio do Ceará

Rua Eliseu Uchoa Becco, 600

Bairro; Água Fria

CEP: 60810-270 - Fortaleza - CE - Brasil

E-mail: richellyrocha@hotmail.com

\section{Potencial Conflito de Interesses}

Declaramos não haver conflito de interesses pertinentes.

Apoio

Programa de Iniciação Científica do Centro Universitário Estácio do Ceará. 